\title{
Correlation between student collaboration network centrality and academic performance
}

\author{
David L. Vargas, ${ }^{1}$ Ariel M. Bridgeman, ${ }^{1}$ David R. Schmidt, ${ }^{1}$ Patrick B. Kohl, ${ }^{1}$ \\ Bethany R. Wilcox, ${ }^{2}$ and Lincoln D. Carr ${ }^{1}$ \\ ${ }^{1}$ Department of Physics, Colorado School of Mines, Golden, Colorado 80401, USA \\ ${ }^{2}$ Department of Physics, University of Colorado Boulder, Boulder, Colorado 80309, USA
}

(Received 1 August 2018; published 15 October 2018)

\begin{abstract}
We compute nodal centrality measures on the collaboration networks of students enrolled in three upperdivision physics courses, usually taken sequentially, at the Colorado School of Mines. These are complex networks in which links between students indicate assistance with homework. The courses included in the study are intermediate classical mechanics, introductory quantum mechanics, and intermediate electromagnetism. By correlating these nodal centrality measures with students' scores on homework and exams, we find four centrality measures that correlate significantly with students' homework scores in all three courses: in-strength, out-strength, closeness centrality, and harmonic centrality. These correlations suggest that students who not only collaborate often, but also collaborate significantly with many different people tend to achieve higher grades. Centrality measures between simultaneous collaboration networks (analytical versus numerical homework collaboration) composed of the same students also correlate with each other, suggesting that students' collaboration strategies remain relatively stable when presented with homework assignments targeting different skills. Additionally, we correlate centrality measures between collaboration networks from different courses and find that the four centrality measures with the strongest relationship to students' homework scores are also the most stable measures across networks involving different courses. Correlations of centrality measures with exam scores were generally smaller than the correlations with homework scores, though this finding varied across courses.
\end{abstract}

DOI: 10.1103/PhysRevPhysEducRes.14.020112

\section{INTRODUCTION}

Physics education research has enjoyed a great deal of success in identifying and clarifying students' difficulties with physics concepts, developing problem-solving methods, and structuring the knowledge that is taught to students [1-5]. Such studies have allowed researchers to make quantitative statements about the presence and persistence of students' difficulties, in contrast to historical physics education that relied on anecdotal information [2]. In recent years, physics education researchers have begun taking advantage of another powerful tool for quantitative analysis in the social sciences known as complex network theory [6,7], the use of which is often referred to as social network analysis (SNA). SNA has a significant history of use in educational research generally [7-9]; however, the use of SNA has only recently begun to gain traction within the physics education research community (see, e.g., Refs. [10,11]).

Published by the American Physical Society under the terms of the Creative Commons Attribution 4.0 International license. Further distribution of this work must maintain attribution to the author(s) and the published article's title, journal citation, and DOI.
In this paper, we use SNA to study self-reported student collaboration and its potential relation to student performance. We examine how collaboration between students evolves between semesters and how nodal centrality measures correlate with homework versus exam grades. Furthermore, we compute the differences in correlation strengths between our measures, allowing us to quantify which measures are most strongly related to student grades. Finally, we also compare the network centrality of students between the collaboration networks of different types of homework assignments within a single course, allowing us to assess the similarity of roles adopted by students in response to assignments of differing nature.

Complex network measures provide succinct summaries of the order present in complex networks. Often such measures are aggregate summaries of the entire structure of a network and are useful because the structure of connectivity can determine the efficiency of processes taking place in the network as observed in social, neural, communication, and transportation networks [7,12]. Furthermore, the nodal centrality measures we review in Sec. IV tell us how well connected students are in the context of their homework collaboration networks, as well as how those connections can serve different purposes within the network $[13,14]$. For example, a simple measure of one way 
a student can be connected to other students in the network is encapsulated by their out-strength. Outstrength is simply the number of peers a student helps with homework and, thus, is one measure of the influence of a student in a collaboration network. Parallel to outstrength is in-strength, the number of students that help a particular student with homework. While out-strength can be thought of as a coarse measure of the influence of a student on the collaboration network, in-strength is a measure of how a student gathers information from different parts of the network. Other more subtle measures of a student's connections within the network include closeness centrality and the related harmonic centrality, both of which look at the "distance" between students (i.e., if information is to pass from student $i$ to student $j$, how many other students must it go through first), as well as betweenness centrality, which looks at the degree to which a particular student can control the flow of information between other students. These centrality measures as well as several others are discussed in detail in Sec. IV. As described in that section, each of these various network centrality measures provide us with different perspectives on various roles a student can play with respect to the flow of information within the collaboration network. Together, the correlations of these different centrality measures with student grades provide insight into how strongly and in what manner different collaboration patterns are central to the educational process.

While the application of complex network-based methods to student networks formed within the physics classroom are rare, there is a growing body of work in this area. In particular, network analysis has been consistently applied to investigations of student persistence [15-18]. The use of network analysis in the context of understanding student persistence is motivated by the idea that a student's decision to persist (or not) within a particular major is impacted by their integration within, and interaction with, their academic and social communities [16]. Forsman et al. reported on students' in-class social and academic interactions. They showed that these social and academic networks had distinct connection patterns indicating that the underlying processes governing the formation of these networks is different. They then argued that both networks need to be considered when addressing student persistence. Zwolak et al. [16] created networks based on students' inclass interactions within a highly interactive introductory course and found that certain network centraility measures correlated significantly with students' persistence into the next course in the sequence. This correlation held even when controlling for the impact of a student's grade. Zwolak et al. [17] later built on this work by incorporating an additional network based on students' interactions outside of class. They found that for middle-performing students, out-of-class centrality measures dominated in terms of predicting students' persistence to the next course in the sequence.

\section{A. Prior research with social network analysis}

Network analysis has also been utilized in investigations not directly tied to student persistence. Dou et al. [19] reported correlations between network centrality measures and changes in students' self-efficacy over the course of an introductory physics course taught using modeling instruction. Brunn and Brewe [11] used network centrality measures calculated based on students' interactions in an introductory physics course to predict their grades in a future course. The use of network analysis in physics education research has also been extended beyond social networks. For example, Bodin [10] applied network analysis to visualize connections between students' epistemic ideas when solving physics problems involving simulations and modeling tasks, and Brewe et al. applied network analysis to characterize students' responses to an introductory conceptual assessment.

The current study is distinct from the work described above in several ways. The majority of the prior work focuses on students' in-class interactions in the context of an introductory physics course utilizing a highly interactive curriculum. The current work focuses on three upperdivision courses all taught with a mixture of traditional lecture punctuated by the use of interactive techniques. Additionally, the current work deals with students' out-ofclass collaborations on homework assignments; thus, these interactions are driven almost entirely by the students without the significant pressure to collaborate usually associated with an interactive classroom environment. Using a wide variety of complex network measures, we obtain detailed information about the role of different collaboration strategies in different types of problem sets and on exams. This study is a step towards responding to multiple calls to take advantage of the analysis power of complexity science within physics education research $[17,18]$.

Here, we build off the work described above by addressing the following set of questions. Do wellconnected students have good grades? That is, does access to the reasoning of many of their peers (as determined by their network centrality) better equip students to complete homework assignments, or does excessive participation in a collaboration network stifle the ability of a student to perform well on their own work? Do the benefits of collaboration extend to exams, where a student does not have access to their collaborators? How stable are these measures of collaboration in different contexts? That is, do students tend to take on different roles in response to different types of assignments or different subject matters, or are students' collaboration strategies static?

\section{DATA COLLECTION}

The Colorado School of Mines (Mines) is a public research university in Golden, Colorado. The university, which has close to six thousand undergraduate and 
Fall 2012

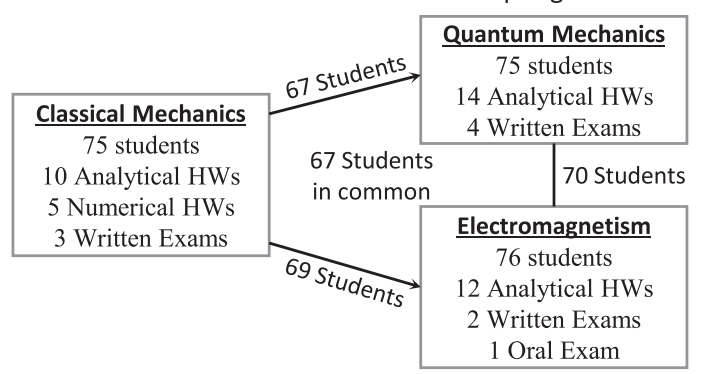

FIG. 1. Course information for classical mechanics, quantum mechanics, and electromagnetism. Lines connecting two courses indicate the number of students common to both courses. There were 67 students enrolled in all three courses.

graduate students, focuses on engineering and the applied sciences. Additionally, it is one of very few institutions that awards more than 50 Physics Bachelors per year, placing it in the top ten of all Ph.D. granting departments in the U.S. [20]. The physics department has research focus areas in condensed matter, subatomic, optical, renewable energy, theoretical, and computational physics.

The data for our networks were collected over two semesters: Fall 2012 and Spring 2013. Students in their junior year in engineering physics at Mines take Cclassical mechanics during the Fall semester and both quantum mechanics and electromagnetism during the spring semester. The course in classical mechanics covers Lagrangian and Hamiltonian mechanics. The electromagnetism course is the first course in a two-course sequence, and covers electrostatics and magnetostatics, including the appropriate Maxwell equations, boundary conditions, and treatments of free and bound sources. The course in quantum mechanics introduces the formalism of quantum mechanics (e.g., the solutions of a particle in a box, scattering from a potential well, etc.). We summarize the homework, exam, and enrollment information for the three courses in Fig. 1. Note that the longitudinal nature of our data means that many students in our data set appear in all three courses. Prior to their junior year at Mines, physics majors are encouraged to collaborate in a physics studio setting, a setting in which students work in groups of three to complete homeworklike assignments and labs. Additionally, the summer before their junior year, Mines physics majors participate in a physics field session in which groups of ten students move between sections on computing, vacuum systems, machining, and lasers. In all of these sections, students are encouraged to collaborate, and in some of them students are split into groups of three to complete assignments. Thus, collaboration is an explicit part of Mines' lower-division program already, and is strongly encouraged.

There were two forms of data collection: paper forms during Fall 2012 and electronic spreadsheets during Spring 2013. In the paper form of data collection, students were provided with a form for each homework assignment in which they were to self-report any students they helped or received help from for each assigned problem when working on the homework outside of class. In the electronic form of data collection, students provided the same data by entering the names of their homework collaborators into question or answer boxes on the learning management system Blackboard [21]. In both cases, students were required to complete the surveys described above in order to receive credit for the associated homework assignments. This policy incentivized survey completion and ensured a nearly complete set of data. Student names were then replaced with a set of randomly generated three letter codes to anonymize the data prior to analysis. We note that data collected on students' interactions was based purely on students' self-reporting on interactions that took place primarily outside of the classroom environment. As such, while the dynamics of those interactions with regards to, for example, collegiality may be an important aspect of the effectiveness of those interactions, the data collected here cannot speak to these aspects of students' interactions.

The data from the surveys above was compared with student grades in the three courses (Fig. 1). The course in classical mechanics had two distinct types of homework assignments-analytical homework assignments $(N=10)$ and numerical homework assignments $(N=5)$-as well as three written exams. For numerical assignments, students were asked to simulate various physical scenarios using Mathematica. For the course in classical mechanics, we computed three measures of a students' performance: the sum of their analytical homework grades, the sum of their numerical homework grades, and the sum of their exam grades. For the courses in quantum mechanics and electromagnetism, all homework assignments were analytical (see Fig. 1), and we measured a students' performance by the sum of their homework grades and the sum of their exam grades. It is important to note that in classical mechanics, the teaching assistants graded exams with subsequent review by the instructor. In quantum mechanics and electromagnetism, the instructors graded all exams themselves. Finally, the instructors of quantum mechanics and classical mechanics both applied curves to exam grades, whereas the instructor of electromagnetism did not.

\section{METHODOLOGY FOR CONVERTING DATA INTO NETWORKS}

From the data collected in the surveys above, we constructed directed and weighted networks for each course and type of homework assignment using the following procedure. A network is a collection of nodes and links. Nodes are any object that can be connected to any other object by some relation and links are the connections between nodes. In our networks, nodes correspond to students, and a link corresponds to an interaction between the students consisting of providing or receiving assistance. Note that this is not 
a symmetric relation; if $i$ helped $j$, it does not imply that $j$ helped $i$. This is the defining feature of a directed network; its connections are asymmetrical. For directed networks, one says that a link goes from node $i$ to node $j$ to indicate the direction of the link. For the $k$ th network a link is placed from node $i$ to node $j$ if and only if (iff) student $i$ helped student $j$ with homework assignment $k$. Summarizing our network in terms of the entries of an adjacency matrix,

$$
A_{i j}^{k}= \begin{cases}1 & \begin{array}{l}
\text { iff student } i \text { helped student } j \\
\text { with homework assignment } k
\end{array} \\
0 & \text { otherwise }\end{cases}
$$

However, we found that it was necessary to resolve discrepancies in the reports provided by students. For example, student $i$ may claim that they helped student $j$,

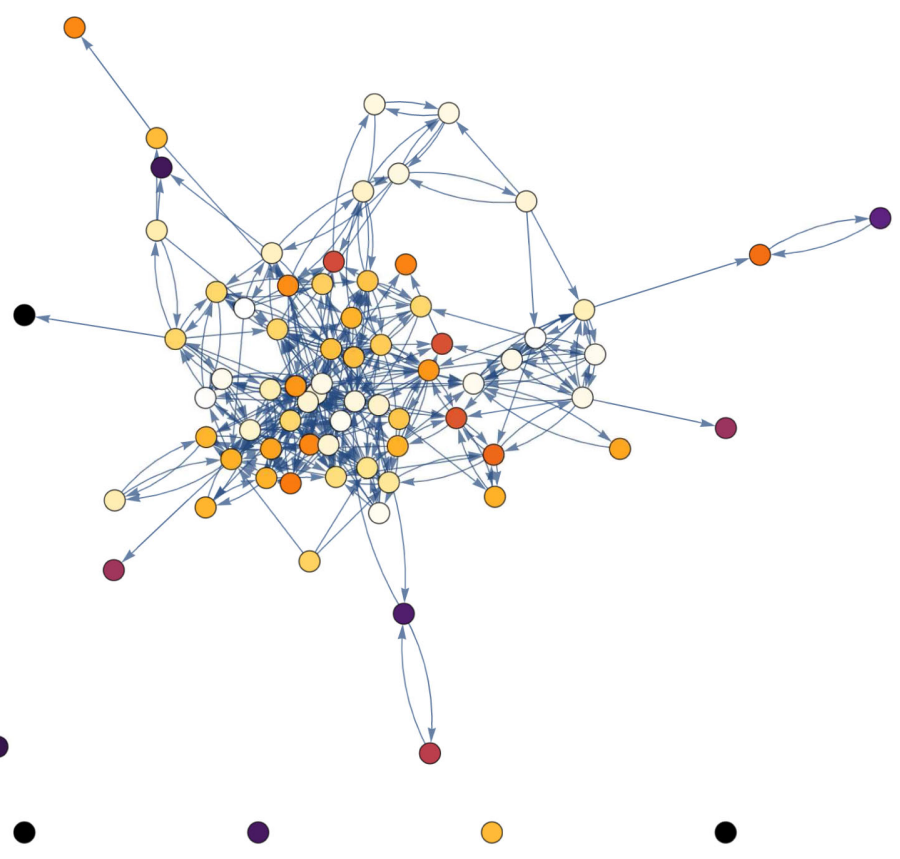

(b) Numerical Assignments Network for Classical Mechanics

(a) Analytical Assignments Network for Classical Mechanics

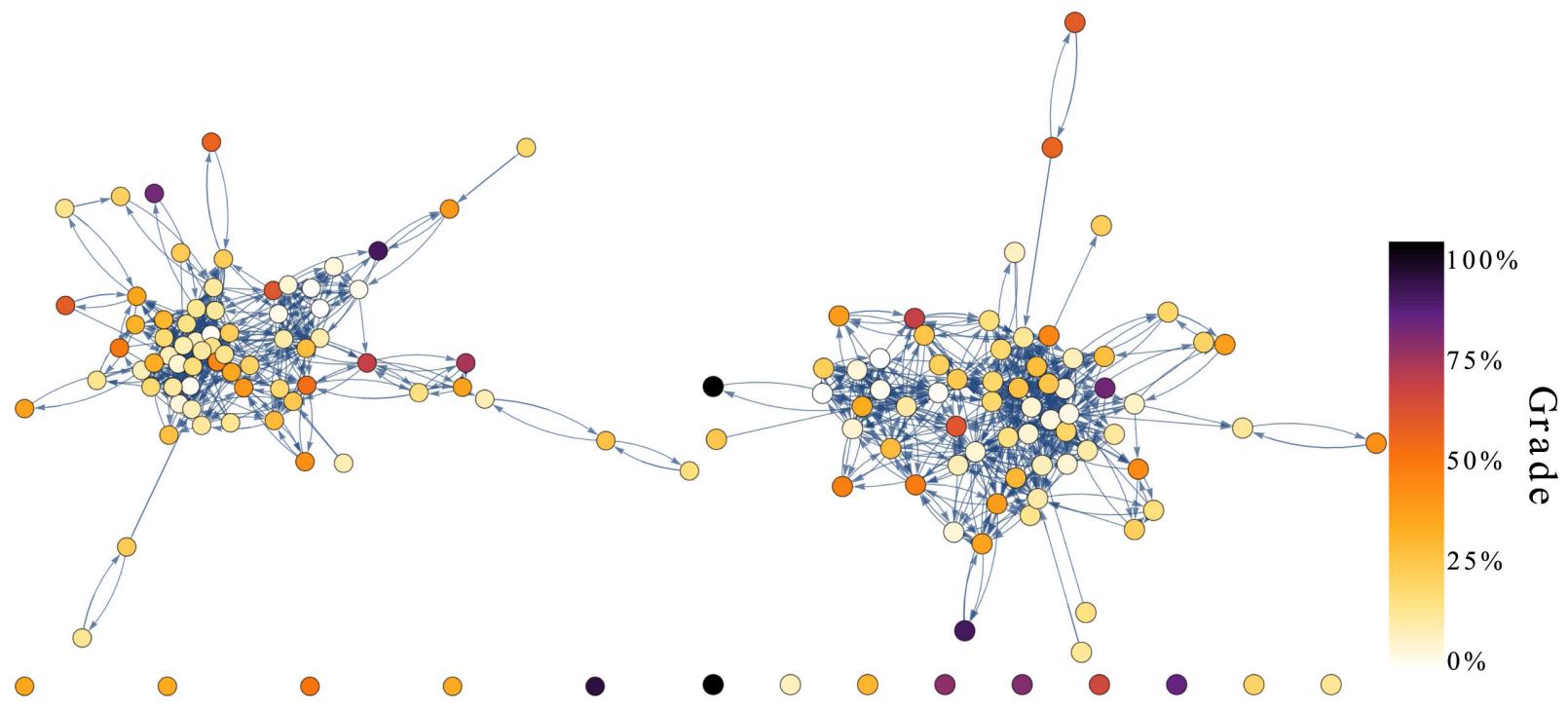

(c) Assignments Network for Quantum Mechanics (d) Assignments Network for Electromagnetism

FIG. 2. Student collaboration networks for three upper level physics courses. Weighted student collaboration networks constructed from surveys given to students in three upper-division courses: Classical mechanics, quantum mechanics, and electromagnetism. Note that while these networks were generated from the weighted adjacency matrices, for ease of representation, only the direction of links is represented here. Nodes correspond to students and the direction of each arrow indicates the direction of assistance on homework assignments. The color of a node indicates the grade of a student on homework assignments. Although we do not normalize grades in our analysis we present grades as a percentage here to illustrate multiple courses simultaneously. 
but student $j$ 's survey indicates that they did not receive help from student $i$. These discrepancies may be due to forgetfulness or conflicting perceptions of interactions [11]. To resolve the discrepancies in student reports, we employed a Maximal discrepancy resolving technique [22,23]; every reported interaction was considered to have happened as long as one student in the pair reported it (even if the other student did not report it). This maximal discrepancy resolving technique was selected because it resulted in richer and denser adjacency matrices. Discrepancies can occur in either direction of an interaction, and $A_{i j}$ is resolved separately from $A_{j i}$. Other discrepancy sorting cases were investigated but yielded quite sparse adjacency matrices [22,23]. For each course, we then compute a weighted adjacency matrix by summing the adjacency matrices corresponding to the homework assignments in that course,

$$
A_{i j}=\sum_{k=1}^{N_{\text {HW }}} A_{i j}^{k}
$$

where $N_{\mathrm{HW}}$ is the number of homework assignments in the relevant course. Thus if two students $i$ and $j$ collaborated frequently on homework assignments, they will have a heavily weighted connection in one of the weighted networks depicted in Fig. 2. In Fig. 2, nodes are indicated by circles and the links connecting nodes are indicated by the arrows between nodes. The direction of the arrow indicates the direction of assistance. For the course in classical mechanics, we construct networks for the numerical homework assignments and the analytical homework assignments separately. This procedure results in two networks for the course in classical mechanics: a network constructed from the collaboration networks on analytical assignments $A_{A}^{\mathrm{CM}}$, and a network constructed from the collaboration networks on numerical assignments $A_{N}^{\mathrm{CM}}$. We denote the network for the course in quantum mechanics by $A^{\mathrm{QM}}$, and the network for the course in electromagnetism as $A^{\mathrm{EM}}$.

\section{COMPLEX NETWORK ANALYSIS}

Using the NetworkX network analysis software [24], as well as some of our own independently developed network analysis code, we study the networks described in the previous section by computing various nodal centrality measures and other measures of the structure of a node's connections. We then compute the correlation between these measures and different estimators of student performance. Nodal centrality measures are measures of a node's importance and role within the structure of the network. As they are quantitative measures of each student's role in the collaboration network, nodal centrality measures are ideal for our study. We selected eight standard nodal centrality measures which have been used previously in the context of complex network analysis [13,14,16-18]—out-strength, in-strength, net out-strength, out-disparity, in-disparity, local clustering, closeness centrality, harmonic centrality, and betweenness centrality. Each of these centrality measures was selected for both its prevalence in the literature and our own sense of its potential relevance within our collaboration networks. Below, we discuss each of these centrality measures in detail including our motivation for selecting each.

The out-strength of a node is the sum of its outgoing connections to other nodes and is defined as

$$
s_{i}^{\text {out }}=\sum_{j=1}^{L} A_{i j},
$$

where $L$ represents the number of nodes (i.e., students) in the network. A node can have high out-strength if it has outgoing connections to many other nodes, or if it has strong connections to only a few other nodes. Stated simply, students who help many of their peers and students who frequently help a smaller set of peers both can have a high out-strength. One might anticipate that students with high out-strength will have higher grades either because they are students who already have a strong grasp of the material or because they have many opportunities to explain their reasoning to other students.

The in-strength is similarly defined and distinguishes a node by the number of incoming connections

$$
s_{i}^{\text {in }}=\sum_{j=1}^{L} A_{i j}^{T},
$$

or the number of instances in which a student received help. In-strength is included as a comparison to out-strength as one might expect that students with high in-strength are students who are already struggling in the course and thus might achieve lower grades on average.

We also study the net out-strength

$$
s_{i}^{\text {net }}=s_{i}^{\text {out }}-s_{i}^{\text {in }} .
$$

Students with high net out-strength correspond to students that help many other students but are not helped by many students. This measure adds to the comparison of instrength and out-strength by distinguishing between students who both give and get help an equal amount from those who either give or get help with more frequency. Again, we might expect that students with high net outstrength are stronger students overall and might naturally achieve better grades.

The out-disparity of a node's connections is a measure of the nonuniformity of the outgoing connection strengths. If a node has a single strong connection in addition to other, much weaker connections, the node has high out-disparity. If the connection strengths of a node are all approximately equal strength, then it has a low out-disparity. Out-disparity is defined as $[25,26]$ 


$$
Y_{i}^{\text {out }} \equiv \frac{1}{\left(s_{i}^{\text {out }}\right)^{2}} \sum_{j=1}^{L}\left(A_{i j}\right)^{2}=\frac{\sum_{j=1}^{L}\left(A_{i j}\right)^{2}}{\left(\sum_{j=1}^{L} A_{i j}\right)^{2}}
$$

Nodes with high disparity correspond to students that collaborate with certain nearest neighbors much more often than they collaborate with other nearest neighbors. Nodes with low disparity correspond to students that collaborate equally with all students that they collaborate with. Analogously, in-disparity measures the nonuniformity of the incoming connection strengths. To compute $Y_{i}^{\text {in }}$ one makes the substitution $A \rightarrow A^{T}$ in Eq. (6), resulting in

$$
Y_{i}^{\text {in }} \equiv \frac{1}{\left(s_{i}^{\text {in }}\right)^{2}} \sum_{j=1}^{L}\left(A_{i j}^{T}\right)^{2}=\frac{\sum_{j=1}^{L}\left(A_{i j}^{T}\right)^{2}}{\left(\sum_{j=1}^{L} A_{i j}^{T}\right)^{2}} .
$$

Note that out-disparity can only be defined for student's with $s_{i}^{\text {out }}>0$; thus students with $s_{i}^{\text {out }}=0$ are not included in correlations involving out-disparity. The same holds for in-disparity for student with $s_{i}^{\text {in }}=0$. A student with high out- and/or in-disparity represents someone who does not send or receive information broadly from the network. One might predict that such students would have less access to the reasoning of their peers and thus potentially achieve lower scores than more broadly connected students.

The local clustering coefficient is a measure of the transitivity of connections of individual nodes, that is, the likelihood that $a$ is connected to $c$, given that $a$ is connected to $b$ and $b$ is connected to $c$. The local clustering coefficient is only defined on undirected networks. Before computing the local clustering coefficient via the equation below, we first convert our directed networks into undirected networks as such that, $A_{i j}^{\text {undirected }}=\max \left(A_{i j}, A_{j i}\right)$ :

$$
c_{i}^{L} \equiv \frac{T(i)}{k_{i}\left(k_{i}-1\right)},
$$

where $T(i)$ is the number of existing triangles in which node $i$ is a vertex, and $k_{i}$ is the degree of node $i$. Effectively, this provides the fraction of all possible triangles through node $i$ that actually exist. Nodes with low local clustering correspond to students whose collaborators do not tend to collaborate with each other. Nodes with high local clustering correspond to students whose collaborators frequently collaborate with each other, such as in tight-knit study groups. Study groups might provide productive stability and community that could enhance students' scores but might also restrict students from broadly collaborating with many other students.

In a weighted network one can define a distance between any pair of nearest-neighbor nodes. For our analysis, we define the distance between nearest neighbors $i$ and $j$ to be the inverse of the weight connecting them

$$
D_{i j}=\frac{1}{A_{i j}} .
$$

If nodes $i$ and $j$ are not directly connected by a link then $D_{i j}=\infty$. This definition of the distance between nearestneighbor nodes is then used to define the shortest-path distance between any two nodes $d_{i j}$. A path connecting node $i$ to node $j$ is a sequence of links along which one may walk to traverse the network from node $i$ to node $j$ when one walks along links in the direction of the link. The shortest-path distance between two nodes is the sum of the nearest-neighbor weights $D_{i j}$ along the shortest path connecting two nodes, that is,

$$
d_{i j}=\min _{P} \sum_{(l, k) \in P} D_{l k},
$$

where $P$ is a path connecting node $i$ to node $j$. In this way, the shortest path distance represents a measure of how easily information can pass between two students in the network. Shortest path distance is used as a basis for the remaining three centrality measures.

Closeness centrality is a measure of how close a node is on average to other nodes when one must travel along directed links in the direction of the link. Closeness centrality is defined as

$$
c_{i}^{C}=\frac{n-1}{|A|-1} \frac{1}{\sum_{j \neq i} d_{i j}},
$$

where $n$ is the number of nodes reachable from node $i$, and $|A|$ is the number of nodes in the network defined by the adjacency matrix $A[14,24]$. Reachable means that one can travel from node $i$ to node $j$ by walking along links in the direction of the link. Any nodes that are not reachable from node $i$ are neglected in the sum of Eq. (11). In the context of social networks, closeness centrality can be thought of as a measure of independence as described in Ref. [14]. This is because a node with a large closeness centrality does not have to rely on any one or two other nodes to transmit messages across the network [14]. In the context of weighted student collaboration networks, closeness centrality is a measure of both the frequency with which a student assists others and how widely a student collaborates. Students with high closeness centrality have strong connections with many other students and, as a result, access to much of the information present in the network; thus, these students might exhibit higher performance.

Harmonic centrality is also a measure of how close a node is to other nodes in the network when one must travel along directed links in the direction of the link. Harmonic centrality is defined as 


$$
c_{i}^{H}=\sum_{j \neq i} \frac{1}{d_{i j}}
$$

where $d_{i j}$ is the shortest path distance from node $i$ to $j$ $[24,27]$. Harmonic centrality has a similar definition to closeness centrality, both being defined in terms of the inverse distances between nodes. The intuition for the two measures is the same. Nodes that are close to other nodes are more central as measured by closeness centrality and harmonic centrality. However, when computing harmonic centrality, if node $j$ is not reachable from node $i$, then the distance between the two nodes is set to $d_{i j}=\infty$. The corresponding term in the sum is then set to zero, $1 / d_{i j}=1 / \infty \equiv 0$. This may be preferable to the procedure used to calculate closeness centrality as this procedure has been shown to introduce a bias towards nodes in small components because it does not take into account nodes that are not reachable by the node of interest [27].

Betweenness centrality is measure of how important a node is as a go-between for message transmission between nodes in a network, assuming that information travels along paths of shortest distance [28]. Betweenness centrality is defined as

$$
c_{i}^{B}=\sum_{j, k \in V} \frac{\sigma(j, k \mid i)}{\sigma(j, k)},
$$

where $\sigma(j, k \mid i)$ is the number of shortest paths from node $j$ to node $k$ that pass through node $i$ and where $\sigma(j, k)$ is the number of shortest paths from node $j$ to node $k$ [13,24]. Nodes with high betweenness centrality correspond to students with the most control over information transfer throughout the network. Therefore, the weight of the links in our networks do not modify betweenness centrality directly, but do indirectly contribute through the path lengths. Students with high betweenness centrality represent links between different sections of the network and thus help to move information through the network. Again, this access to the reasoning of different sections of the network might result in higher performance.

Together, the nine centrality measures described above give information about students' collaboration strategies and patterns, as well as their role in the movement of information throughout the network. In the next section, we correlate these measures with both homework and exam scores to identify which centrality measures are most closely linked to students' performance.

\section{RESULTS}

We now correlate each of the nodal centrality measure described in the previous section with students' homework assignment or exam scores. In Fig. 3, we display the results of these calculations. Statistical significance of the correlation coefficients for each course and assignment type was determined at the $p<0.05$ level using Holm-Bonferroni corrected $p$ values calculated via a bootstrap resampling with 10000 resamplings of each correlation coefficient [29]. The use of bootstrap resampling was motivated by the fact that centrality measures from complex network analysis are inherently interdependent and, thus, violate the assumption of independence fundamental to standard parametric statistics [15].

\section{A. Do well connected students get good grades?}

With respect to our first question of interest-do well connected students get good grades?-Fig. 3 suggests that this depends on both the type of centrality measure and the course in question. Four centrality measures have statistically significant correlations to homework grades (both numerical and analytical) for all three courses: closeness centrality, harmonic centrality, in-strength, and outstrength. Recall that in- and out-strength measure how often a student collaborates by receiving or giving help, respectively, while closeness and harmonic centrality are both measures of how "far" a student is from other students. Figure 3 shows that in- and out-disparity have a negative correlation to homework scores in all classes and that correlation is statistically significant for at least one type of homework (analytical or numerical) in two of the three courses. In- and out-disparity measure a student's tendency to collaborate often with only a small number of students. This result, combined with the significant positive correlations with the four centrality measures above suggest that students who not only collaborate often, but also collaborate significantly with many different people tend to achieve higher grades.

Figure 3 also shows three measures that tend to have smaller, and less often statistically significant, correlations with homework scores; these are betweenness centrality, local clustering, and net out-strength. Recall that betweenness centrality measures the extent to which a student can control the flow of information between other students, local clustering determines the extent to which a student may be part of a close knit study group, and net out-strength determines whether a student helps others more than they are helped. That these measures are less often significant suggests that collaborating often and widely has a greater relation to homework grades than being part of a single close knit study group or being a link between groups or students. The small correlation of homework scores with net out-strength relative to the correlations for both instrength and out-strength also suggests that the quantity of collaborations matters more than the type of collaboration (i.e., helping others versus being helped). This finding is perhaps surprising given that one might expect that students who more often give help than receive it would be the naturally higher performing students.

Comparing the correlations in Fig. 3 across the three courses shows similar trends across most of the measures. 


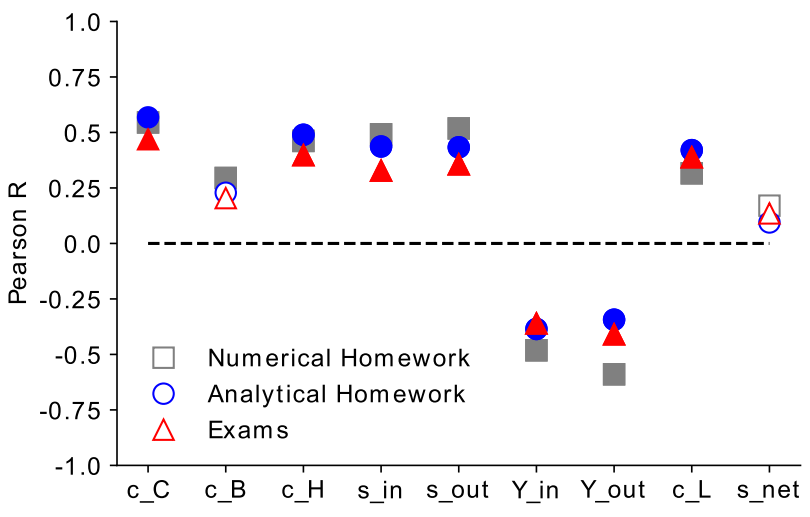

(a) Classical Mechanics Analytical Network

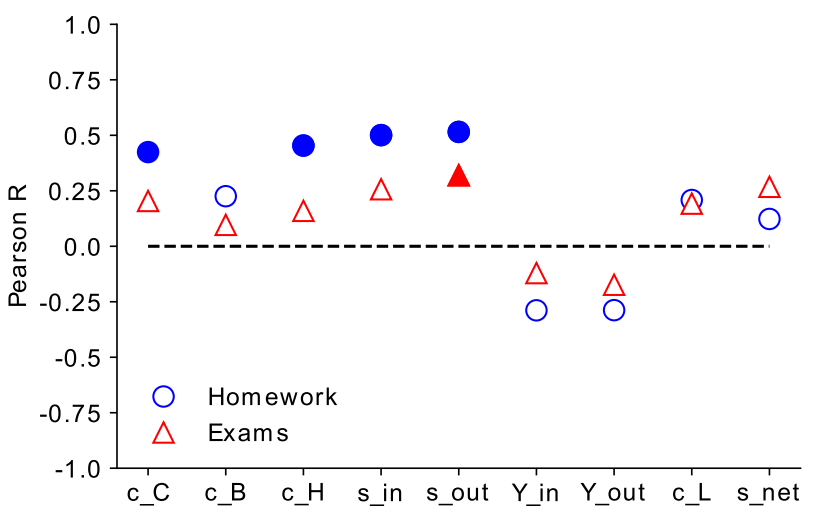

(c) Quantum Mechanics Network

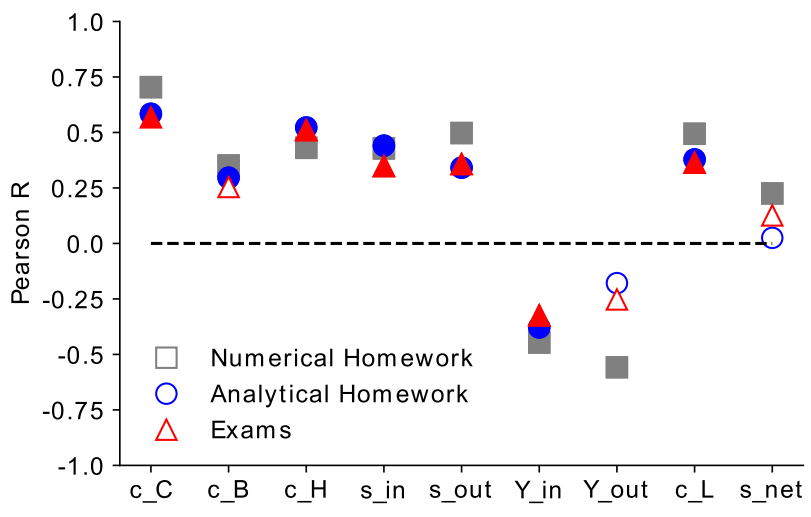

(b) Classical Mechanics Numerical Network

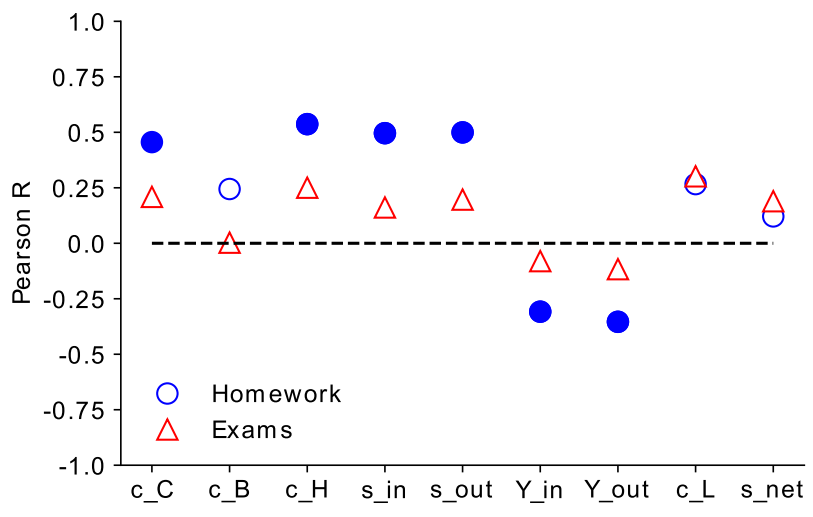

(d) Electromagnetism Network

FIG. 3. Correlation of nodal centrality measures with student grades in three upper level physics courses. Correlation of complex network measures with student grades for three courses: classical mechanics, quantum mechanics, and electromagnetism. Filled markers indicate correlation coefficients that are statistically significantly different from a correlation coefficient of 0 (Holm-Bonferroni corrected $p<0.05$ ). Statistical significance was determined using a bootstrap resampling with 10000 resamplings of each correlation coefficient.

One significant exception to this is the local clustering coefficient which is larger (and statistically significant) in both classical mechanics networks than in the networks for the other two courses. This suggests that for the classical mechanics course, integration into well-established study groups had a stronger relation to students' scores on both the analytical and numerical homework. To better understand possible sources of this difference, we look at differences between the courses themselves. classical mechanics is the first "hard" upper division course most physics students take; in fact, it is generally considered by students to be one of the hardest courses in the Mines physics curriculum. Thus, students may still be modifying their lower-division study habits to accommodate this additional challenge. Thus, we may be seeing the effect of tight knit study groups in a way that dissipates as students get to know each other in the ensuing upperdivision courses. The numerical component of the classical mechanics course, which was not present in the other courses and is unique to the curriculum, also represents a possible contributing factor.

\section{B. Does the benefit of collaboration extend to exams?}

Another question of interest for this study pertains to whether the benefits of collaboration extend beyond homework scores to students' performance on exams. It may be that the individual nature of exams suppresses the impact of collaboration on students' performance. Here again, Fig. 3 suggests that the answer depends on what course we look at. For both quantum mechanics and electromagnetism, correlations between all centrality measures and exam scores are lower than correlations of the same measure with homework scores. In fact, only out-strength correlates significantly with exam scores and then only in quantum mechanics. This suggests that for these courses, the potential benefit of centrality within the collaboration network does not extend to exams. On the other hand, 
in the classical mechanics course, the correlations of centrality measures with exam scores is comparable to the corresponding correlations for homework scores for nearly all measures. Beyond the inclusion of a fairly significant conceptual component in the classical mechanics exams ( $\sim 1 / 4$ of the questions), it is not obvious why the classical mechanics networks appear to correlate better with exam scores. However, these findings suggest that whether the benefits of collaboration extend to exam scores as well as homework scores depends on the structure of the course and/or content of the exams. Application of this analysis to other courses will be necessary to pinpoint the course and exam features that best realize the benefits of students' collaboration. It is worth noting that, depending on the instructional goals of the course, exams may not be designed to realize the benefits of student collaboration. Moreover, all exams in this study were traditional, individual exams; these findings would likely shift if the format of the exam was less traditional (e.g., group exams).

\section{How stable are collaboration strategies across assignments and contexts?}

In our study, we have two simultaneous networks composed of exactly the same students: the analytical and numerical networks for classical mechanics. This allows us to investigate another question of interest-how stable are these centrality measures across different types of assignments? Comparing across the two classical mechanics networks (analytical and numerical), we see in Fig. 3 nearly identical patterns in the correlations of the different centrality measures with homework scores both in terms of magnitude of the correlations and which correlations are statistically significant. We also see very similar correlations when correlating network centrality measures created using information from the analytical networks to the scores on the numerical homework and vice versa. Together these findings suggest that these centrality measures are quite stable across different types of homework. We can also use these two simultaneous networks to quantify the stability of the roles taken by students in response to different types of homework assignments. To do this, we correlated centrality measures calculated using the analytical network with those calculated using the numerical network. We found large correlations (in this case $r>0.5$ ) for eight of the nine centrality measures [see Fig. 4(a)]. Only the local clustering coefficient had only a moderate correlation $(r=0.33)$ across networks. All correlations were statistically significant (bootstrap resampling and Holm-Bonferroni corrected $p<0.05)$. This result suggests that students' collaboration strategies remain relatively stable when presented with different types of homework assignments.

In our study, we also have a large subset of students who took all three courses $(N=67$, see Fig. 1). Focusing specifically on these students, we can also investigate the stability of network centrality measures across time as the students advance from classical mechanics to quantum mechanics and electromagnetism. Since all of these courses are upper-division (typically junior-level) courses, these students have already had much of their undergraduate career to develop collaboration strategies that they believe work for them; thus, we might anticipate that their strategies would be relatively stable over time and across courses. In Fig. 4(b), we correlate each centrality measure, student by student, between classical mechanics and quantum mechanics and also between classical mechanics and electromagnetism. Since neither quantum mechanics nor electromagnetism included numerical homework, we utilize the classical mechanics analytical network for the purposes of investigating stability between these courses. Of the nine network centrality measures,

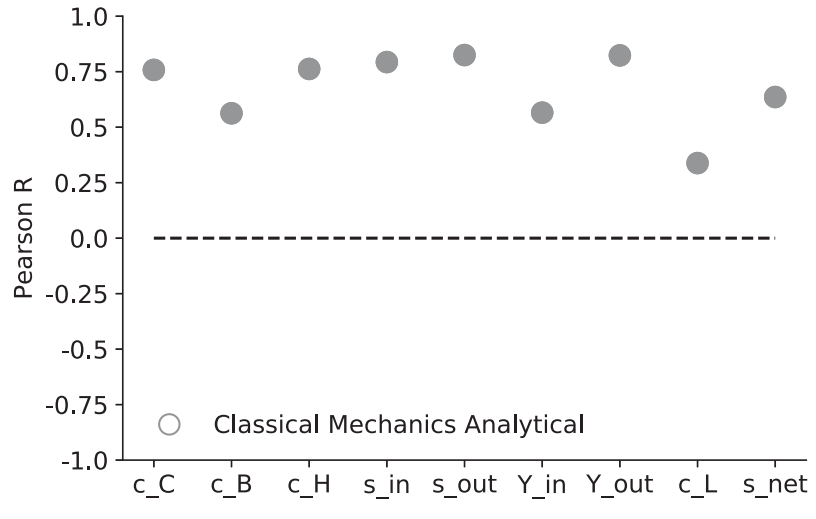

(a)

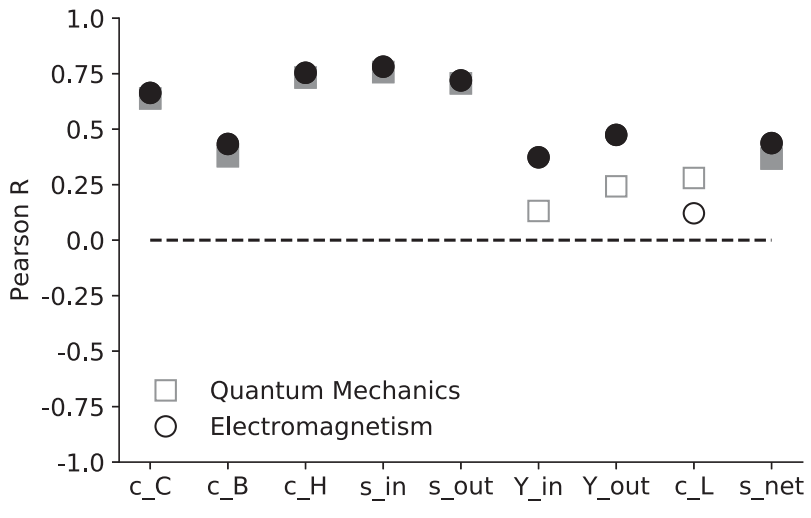

(b)

FIG. 4. Correlation of nodal centrality measures from the classical mechanics analytical network with (a) the corresponding nodal centrality measure from the classical mechanics numerical network, and (b) the corresponding nodal centrality measure from the other two courses: quantum mechanics, and electromagnetism. Filled markers indicate correlation coefficients that are statistically significantly different from a correlation coefficient of 0 (Holm-Bonferroni corrected $p<0.05$ ). Statistical significance was determined using bootstrap resampling with 10000 resamplings of each correlation coefficient. 
four showed large correlations [in this case $r>0.6$, see Fig. 4(b)] between the classical mechanics network and both the networks of the other two courses. Interestingly, these are the same four measures with consistent significant correlations with the grades in all three courses (i.e., closeness centrality, harmonic centrality, in-strength, and out-strength). The remaining centrality measures in Fig. 4(b) show smaller correlations suggesting less stability in students' network positions between different course networks. The local clustering coefficient once again highlights as having a particularly small correlation, which in this case is not statistically significant. Thus, the network centrality measures that have the strongest relationship to students' homework scores are also the network centrality measures that appear to be the most stable across networks involving different courses or assignment types.

\section{SUMMARY AND CONCLUSIONS}

We utilized the tools of complex network analysis to form social networks based on students' self-reported collaborations when completing regular homework assignments outside of class in three upper-division physics courses. From these networks, we then calculated multiple network centrality measures which provide information on the collaboration strategies and role of each student with respect to the flow of information within the network. By correlating these nodal centrality measures with students' scores on both homework and exams, we were able to investigate the relationship between students' centrality within the network with their performance in the course to investigate whether particular modes of centrality were more (or less) strongly linked with student performance.

With respect to our first research question-do well connected students achieve higher grades? - we found that four of the nine centrality measures (closeness centrality, harmonic centrality, in-strength, and out-strength) correlated significantly with students' homework scores in all three classes. Moreover, we found two centrality measures (in-disparity and out-disparity) that consistently (though not always significantly) showed negative correlations with students homework scores. Together, the significance of the first four measures, along with the negative correlation from the second two, suggest that students who not only collaborate often but also collaborate significantly with many different people tend to achieve higher homework grades. Additionally, the lack of significance associated with the centrality measure net out-strength suggests that helping others more that one receives help does not have a significant association with performance; in other words, helping others in the network does not appear to be more valuable than being helped with respect to performance. We note that this finding depended on interpreting that all of the centrality measures wholistically; focusing on only one of the measures (e.g., out-strength) could have led to the erroneous conclusions about the importance of helping versus being helped, as well as the importance of broad as opposed to localized collaboration.

With respect to our second research question-do the benefits of collaboration extend to course exams?-we found that correlations of centrality measures with exam scores were generally smaller than the correlations with homework scores, though this finding varied across courses. Note that this finding does not necessarily suggest that exams more accurately represent a student's individual understanding; while the correlation with collaboration is generally smaller, the high stakes nature of exams introduces a number of factors besides ability that can impact a student's exam scores (e.g., stereotype threat).

With respect to our final research question-how stable are collaboration strategies across assignments and contexts?-we also found that students' collaboration strategies are relatively stable when presented with different types of homework assignments (e.g., analytical versus numerical) within the same class. We also found that while some centrality measures appear to shift significantly when students move into a different course, the four centrality measures most strongly related to students' homework scores (closeness centrality, harmonic centrality, in-strength, and out-strength) are also the most stable between networks from different courses.

This work helps provide insight into whether and how students' collaboration impacts their success in the course as measured by course exam and homework grades. It also contributes to a growing body of research utilizing complex network theory to better understand the role of social networks within the undergraduate classroom. However, there are several important limitations to the study. The findings reported here are correlational, and thus, cannot clearly establish that broad collaboration improves students' performance, only that students whose nodal centrality indicates that they collaborate broadly tend to have higher scores. Additionally, these data come from a single institution and have relatively low $N(N<100)$. Replicating these analyses in additional courses at additional institutions will be important for establishing the generalizability of these findings.

For instructors, these results suggest that encouraging students not only to collaborate, but to collaborate with multiple other students may be an effective strategy towards improving students' homework scores. Moreover, they suggest that the benefits of collaboration are not automatically transferred to exam performance, but rather the structure of the course and exams can enhance or suppress the relation between student collaboration and exam scores. Future work could include implementing a similar analysis on collaborations amongst researchers at the graduate level and beyond, using not only in-class studies in graduate school analogous to those considered here, but also collaboration networks on the arXiv. A general hypothesis to be examined is whether an overall broader collaboration 
strategy leads to higher outcomes, in, for example, $h$ index, total number of citations, and grant funding.

\section{ACKNOWLEDGMENTS}

This material is based in part upon work supported by the U.S. National Science Foundation under Grants No. CCF1839232, No. OAC-1740130, and No. PHY-1806372. The authors acknowledge the efforts of Esteban Chavez for developing the code that sorted reporting discrepancies in the survey data we collected. Esteban Chavez was funded under the NSF Grant No. DUE-0836937. We would like to thank Mark Lusk for sharing course data with us. Lastly, we would like to thank all of the students that participated in our study.
[1] L. C. McDermott and E. F. Redish, Resource letter: PER-1: Physics education research, Am. J. Phys. 67, 755 (1999).

[2] L. C. McDermott, Research on conceptual understanding in mechanics, Phys. Today 37, No. 7, 24 (1984).

[3] A. Van Heuvelen, Learning to think like a physicist, Am. J. Phys. 59, 891 (1991).

[4] F. Reif, Scientific approaches to science education, Phys. Today 39, No. 11, 48 (1986).

[5] E. F. Redish and R. N. Steinberg, Teaching physics: Figuring out what works, Phys. Today 52, No. 1, 24 (1999).

[6] S. P. Borgatti, A. Mehra, D. J. Brass, and G. Labianca, Network analysis in the social sciences, Science 323, 892 (2009).

[7] M. E. J. Newman, The structure and function of complex networks, SIAM Rev. 45, 167 (2003).

[8] S. Wasserman and K. Faust, Social Network Analysis: Methods and Applications (Cambridge University Press, Cambridge, England, 1994), Vol. 8.

[9] D. Z. Grunspan, B. L. Wiggins, and S. M. Goodreau, Understanding classrooms through social network analysis: A primer for social network analysis in education research, CBE Life Sci. Educ. 13, 167 (2014).

[10] M. Bodin, Mapping university students' epistemic framing of computational physics using network analysis, Phys. Rev. ST Phys. Educ. Res. 8, 010115 (2012).

[11] J. Bruun and E. Brewe, Talking and learning physics: Predicting future grades from network measures and Force Concept Inventory pretest scores, Phys. Rev. ST Phys. Educ. Res. 9, 020109 (2013).

[12] V. Latora and M. Marchiori, Efficient behavior of smallworld networks, Phys. Rev. Lett. 87, 198701 (2001).

[13] L. C. Freeman, A set of measures of centrality based on betweenness, Sociometry 40, 35 (1977).

[14] L. C. Freeman, Centrality in social networks conceptual clarification, Soc. Networks 1, 215 (1978).

[15] E. Brewe, L. Kramer, and V. Sawtelle, Investigating student communities with network analysis of interactions in a physics learning center, Phys. Rev. ST Phys. Educ. Res. 8, 010101 (2012).

[16] J. P. Zwolak, R. Dou, E. A. Williams, and E. Brewe, Students' network integration as a predictor of persistence in introductory physics courses, Phys. Rev. Phys. Educ. Res. 13, 010113 (2017).

[17] J. P. Zwolak, M. Zwolak, and E. Brewe, Educational commitment and social networking: The power of informal networks, Phys. Rev. Phys. Educ. Res. 14, 010131 (2018).

[18] J. Forsman, R. Moll, and C. Linder, Extending the theoretical framing for physics education research: An illustrative application of complexity science, Phys. Rev. ST Phys. Educ. Res. 10, 020122 (2014).

[19] R. Dou, E. Brewe, J. P. Zwolak, G. Potvin, E. A. Williams, and L. H. Kramer, Beyond performance metrics: Examining a decrease in students' physics self-efficacy through a social networks lens, Phys. Rev. Phys. Educ. Res. 12, 020124 (2016).

[20] AIP Statistical Research Center, http://www.aip.org/statistics.

[21] Blackboard Inc., Blackboard, http://www.blackboard.com.

[22] D. Schmidt, A. Bridgeman, and P. Kohl, Student collaborative networks and academic performance, in APS Meeting Abstracts (2013), http://adsabs.harvard.edu/abs/ 2013APS..APRH15007S.

[23] D. Schmidt, A. Bridgeman, and P. Kohl, Student collaborative networks and academic performance in physics, in PERC 2013 (2013), https://www.compadre.org/per/perc/ 2013/Detail.cfm?id=5129.

[24] A. A. Hagberg, D. A. Schult, and P. J. Swart, Exploring network structure, dynamics, and function using NetworkX, in Proceedings of the 7th Python in Science Conference (SciPy2008), Pasadena, California (2008), p. 11.

[25] S. Boccaletti, V. Latora, Y. Moreno, M. Chavez, and D. Hwang, Complex networks: Structure and dynamics, Phys. Rep. 424, 175 (2006).

[26] E. Almaas, B. Kovács, T. Vicsek, Z. N. Oltvai, and A.-L. Barabási, Global organization of metabolic fluxes in the bacterium Escherichia coli, Nature (London) 427, 839 (2004).

[27] P. Boldi and S. Vigna, Axioms for centrality, Internet Math. 10, 222 (2014).

[28] M. E. J. Newman, Networks: An Introduction (Oxford University Press, Inc., New York, 2010).

[29] L. Wasserman, All of Statistics: A Concise Course in Statistical Inference (Springer, New York, 2004). 